\title{
Intraoperative Cone Beam Computed Tomography of Tracheal Stenting for Stenosis and Fistula Diseases: A Retrospective Cohort Study
}

Jing Li

First Affiliated Hospital of Zhengzhou University

Kaihao Xu

First Affiliated Hospital of Zhengzhou University

Xinwei Han ( $\nabla$ fcchanxw@zzu.edu.cn )

First Affiliated Hospital of Zhengzhou University

Zhaonan Li

First Affiliated Hospital of Zhengzhou University

Yahua Li

First Affiliated Hospital of Zhengzhou University

Xueliang Zhou

First Affiliated Hospital of Zhengzhou University

Juanfang Liu

First Affiliated Hospital of Zhengzhou University

Yuan Yao

First Affiliated Hospital of Zhengzhou University

Zaoqu Liu

First Affiliated Hospital of Zhengzhou University

Dechao Jiao

First Affiliated Hospital of Zhengzhou University

\section{Research Article}

Keywords: CBCT, Tracheal Stent, Airway obstruction, Fistula

Posted Date: February 18th, 2021

DOI: https://doi.org/10.21203/rs.3.rs-206409/v1

License: (c) (1) This work is licensed under a Creative Commons Attribution 4.0 International License.

Read Full License 
Version of Record: A version of this preprint was published at Quantitative Imaging in Medicine and Surgery on January 1st, 2021. See the published version at https://doi.org/10.21037/qims-21-858. 


\section{Abstract}

Background and objectives: Previous experience has shown that angiographic CT is useful in various medical contexts, but little research has been presented regarding the application of Cone beam Computer Tomography (CBCT) in airway stenting. In this study, we retrospectively evaluated the clinical feasibility of CBCT in airway stent placement by conducting a single-center retrospective cohort study.

Methods: A total of 228 patients with stenosis or fistula diseases were treated with metallic airway stents in our hospital from January 1,2015 , to December 31, 2018. CBCT scanning was performed on 100 patients during and after treatment, and the images were compared with those from postoperative CBCT. We also assessed the outcomes and complications in the CBCT-guided and the fluoroscopy-guided group.

Results: Tracheal stenting was performed successfully on the first attempt in 90 patients in the CBCTguided group and 123 patients in the fluoroscopy-guided group. The measured diameters and lengths of the central airway as shown in CBCT were not significantly different from those obtained by MDCT. However, there were significant differences in the visibility of the distal bronchus, pulmonary parenchyma and airway above the upper stent graft; nonetheless, 2 of the 3 anatomical areas were reproduced in a diagnostically relevant way. The rate of major complications was rather low in the CBCT-guided group compared with the fluoroscopy-guided group.

Conclusions: $\mathrm{CBCT}$ images were proven to have sufficient quality to replace MDCT as a reasonable control measure after stent implantation and reduce the complications of airway stent placement when performed in the operation.

\section{Introduction}

With the advancement of interventional instruments and the improvement of operative techniques, tracheal stent implantation has become widely used in the treatment of severe benign and malignant airway stenosis and various types of airway fistula. ${ }^{[1-5]}$ Although stent implantation can significantly alleviate respiratory symptoms in most patients, some studies still note its relatively high complication rate. ${ }^{[6,7]}$ As an important imaging method for preoperative evaluation of lesion location and extent and postoperative evaluation of complications after airway stent placement, multidetector computed tomography (MDCT) takes an essential position in clinical treatment, ${ }^{[8]}$ but this procedure must be performed in a CT laboratory, which inevitably increases the time between the diagnosis of the disease and the corresponding operation and causes a delay in the surgeon's awareness of complications during the operation as well. Cone-beam computed tomography (CBCT), a functional variant of angiographic Carm CT, can quickly provide intraoperative 2- and 3-dimensional reconstructions similar to the CT images. [9] In this way, a 3D scan of the airway can be obtained, and the complications of airway stenting may be reduced. 
Immediate CBCT control after airway stent placement has been performed in our institute in the last five years, and sometimes it has also been used for stent-graft planning in acute or weak patients. Based on the rich experience of interventional radiologists in our institution, we hypothesized that intraoperative CBCT could serve as a suitable replacement for MDCT after stent implantation and, in some cases, for stent-graft planning, while at the same time reducing the complications of airway stent placement and enhancing the surgeon's confidence. Based on this postulate, the purpose of our study was to evaluate the clinical feasibility of CBCT in airway stent placement by conducting a single-center retrospective cohort study.

\section{Materials And Methods}

This retrospective cohort study was approved by the Ethics Committee of Zhengzhou University, and the requirement for informed consent was waived.

\section{Case selection}

The records from January 1, 2015, to December 31, 2018, in the local database were searched manually to identify all patients who had been treated in the western district intervention department of the First Affiliated Hospital of Zhengzhou University for tracheal strictures and fistulas resulting from a heterogeneous group of disorders that usually occur after intubation or surgery. The case selection and grouping process is presented in Fig. 1. Patients who had undergone treatment by airway stenting were eligible for inclusion in the study. Bronchopleural strictures and fistulas were diagnosed on the basis of clinical manifestations and chest CT examination. The airway stenting procedures were performed by four international radiologists (X.-L. Z, Y.-H. L, Z.-N. L, D.-C. J) with 5, 7, 7, and 13 years of intervention experience, respectively. The exclusion criteria were as follows: 1 ) postoperative MDCT images could not be obtained in our PACS (picture and communication system); 2) CBCT reconstruction images were not saved; 3 ) the patient had coagulopathy or abnormal cardiopulmonary function; and 4) the patient could not be followed up as scheduled.

This study was not randomized. Our interventional radiologists rotated daily in the angiography suite only (Toshiba, Aquilion 64, Japan) and were equipped with CBCT technology (Artis zee BA Twin; Siemens AG, Germany; Syngo X-workplace with Syngo CBCT; Siemens AG, Germany). They performed airway stent placement using the available imaging system they were assigned for the day.

\section{Surgical procedures and image acquisition}

All stent placement procedures were performed in a dedicated operating suite- an angiography laboratory equipped or unequipped СВСТ. The detailed steps were similar to those described in previous reports. ${ }^{[1,10]}$ An additional on-table CBCT apparatus was deployed after the stent graft if the angiography laboratory had one. The operator could adjust the position of the stents or introduce a balloon to expand stents through the guide wire when the stent was in an unsuitable location or exhibited incomplete deployment. In some cases, CBCT was performed before the stent graft for stent placement planning. 


\section{Image analysis}

The CBCT image data sets were compared with the posttreatment MDCT images to measure relevant stent diameters and lengths in suitable planes, and the anatomical details were scored. Each image was independently evaluated by 2 experienced interventional radiologists (X.W.H, with 14 years of experience; J.J, with 5 years of experience). The evaluation criteria for the visibility of important anatomic details in the stent graft were derived from the European Guidelines for Quality Criteria for Computed Tomography, ${ }^{[11]}$ which is a 4-point scale: 1 point means that relevant diagnosis is impossible; 2 points means that the visibility is acceptable and that relevant is diagnosis possible; 3 points means a clear reproduction, where diagnosis possible without restrictions; 4 points means perfect imaging.

\section{Postoperative Evaluation and Follow-Up}

The results of imaging follow-up were documented for all patients as a means to assess possible stent migration or underexpansion within three days and then 3 months after the stent placement procedure. We also compared the rate of stent-related complications and the final prognosis for each patient between the fluoroscopy-guided group and the CBCT-guided group.

Technical success was defined as successful placement of the stent in the planned location on the first attempt, as verified on site by fluoroscopy or CBCT. Routine MDCT was performed as a means to assess possible stent migration or underexpansion within 3 days of the procedure and then at 3 months. Each of these follow-up studies was performed irrespective of the planned schedule if warranted by the development of adverse clinical conditions. We also documented telephone follow-ups with patients to track their progress.

\section{Data Analysis}

The measurement data are presented as the mean \pm SD. Categorical data are reported as numbers with percentages in brackets. Comparisons between two groups were conducted by Student's $t$-test, the Wilcoxon signed-rank test for continuous variables and either Pearson's $\chi^{2}$ test or Fisher's exact test for categorical variables. Survival time was calculated according to the Kaplan-Meier method, and a P-value of $<.05$ was considered statistically significant. The variability in relevant measurements and visibility scores between the 2 interventional radiologists was tested using kappa or ICC statistics, and an ICC $\geq$ 0.75 was considered good. The level of agreement for Cohen's kappa is usually defined as follows: less than 0.20 is poor agreement, $0.21-0.40$ is fair agreement, $0.41-0.60$ is moderate agreement, $0.61-0.80$ is good agreement, and more than 0.80 is excellent agreement. ${ }^{[12]}$ All statistical analyses were performed by using statistical software (SPSS 23.0; SPSS, Chicago, II).

\section{Results}

\section{Clinical characteristics and technical success rate of the patient sample}


A total of 228 consecutive patients were enrolled during the period from January 2015 to December 2018; 100 patients received fluoroscopy-guided airway stenting, and 128 patients received CBCT-guided airway stenting. The baseline characteristics of the CBCT-guided group and fluoroscopy-guided group were well balanced (Table 1). Tracheal stenting procedures for stenosis and fistula diseases were successful on the first attempt in 90 patients in the CBCT-guided group and 123 patients in the fluoroscopy-guided group. The technical success rates in these two groups were $90 \%$ and $96 \%(p=.066)$, respectively. In the CBCTguided group, interventional radiologists adjusted incompletely deployed stents in 6 patients to completely adhere. For the remaining 9 patients in both groups, the first attempt at stent placement for stenosis and fistula failed, and the second try was successful. The duration of hospitalization for the CBCT-guided group and the fluoroscopy-guided group was 5 (3-7) days and 7 (3-15) days, respectively $(p=.073)$. Although there was no significant difference, the duration of hospitalization appeared to be shorter in the CBCT-guided group than in the fluoroscopy-guided group.

\section{Comparison of relevant measurements and visibility scores between CBCT and MDCT}

The results are based on the combined data from two independent investigators and are presented in Tables 2 and 3. Only the left and right branch diameter measurements showed significant differences between $\mathrm{CBCT}$ images and MDCT images. When we compared the visibility scores of the anatomical areas in the two types of images, significant differences were observed in the visibility of the distal bronchus, pulmonary parenchyma and airway above the upper stent graft. However, 2 of the 3 anatomical areas were reproduced in a diagnostically relevant way, defined as a visibility score of 2 or better.

\section{Interobserver variation}

A summary of interobserver variability between the two investigators is shown in Table 4 and Table 5 in Supplementary Information. In assessing relevant measurements, the overall agreement was almost perfect $(I C C=0.812-0.998)$. For all anatomical areas, we observed a rather high degree of interobserver agreement; the overall agreement was moderate $(k=0.652-0.772)$, except for $B$ (assessing the numbers and positions of markers in the stent graft), for which almost perfect agreement $(k=0.824)$ was seen.

\section{Complications}

No procedure-related death, choking, stent rupture, severe bleeding, prolonged hypoxia, or airway perforation occurred in any patient during stent insertion or removal. Minor complications in the CBCTguided group included slight airway hemoptysis, chest or throat pain, laryngeal foreign body sensation, and eating disorder, and the corresponding incidence rates were $7 / 100$ (7\%), 4/100 (4\%), 2/100 (2\%), and $1 / 100(1 \%)$, respectively, while the incidence rates in the fluoroscopy-guided group were $9 / 128(7 \%)$, $5 / 128(4 \%), 2 / 128(2 \%)$ and $3 / 128(2 \%)$, respectively $(p>0.05)$. There was a significant difference in the incidence of major complications $(p=.019)$ between the two groups, especially for stent migration ( $p$ $=.019$ ), which was less common in the CBCT-guided group than in the fluoroscopy-guided group (Table $6)$. 


\section{Outcome and follow-up}

For the CBCT-guided group and the fluoroscopy-guided group, the median follow-up durations were 51 months (range 11-60 months) and 45 months, respectively (range 13-60 months), respectively. By the time of the data collection, 38 patients $(38.0 \%)$ and 51 patients $(43.0 \%)$, respectively, had died. There were 47 patients (47\%) in the CBCT-guided group and 51 patients $(39.8 \%)$ in the fluoroscopy-guided group who showed improvements in clinical symptoms, and it was confirmed that $12(12 \%)$ and $19(14.8 \%)$ patients, respectively, were cured as determined by MDCT. Three patients (3\%) and seven patients (5.4\%), respectively, were lost to follow-up. None of these variables showed a significant difference between the two groups (Table 7). The 1-, 3- and 5-year overall survival rates of the CBCT-guided group were 100.0\%, $72.4 \%$ and $61.0 \%$, respectively, while those of the fluoroscopy-guided group were $100.0 \%, 69.8 \%$, and $57.0 \%$, respectively ( $p>05$ ) (shown in Fig. 2).

\section{Discussion}

Measurements of the diameters and lengths of the central airway based on CBCT images were not significantly different from those obtained by MDCT. Although there were significant differences between CBCT and MDCT in the visibility of the distal bronchus, pulmonary parenchyma and airway above the upper stent graft, 2 of the 3 anatomical areas were reproduced in a diagnostically relevant way, which resulted in low scores on $\mathrm{CBCT}$ images for these objects that, in some cases, tended to be outside the imaging field. ${ }^{[13]}$ Therefore, our results indicate that $\mathrm{CBCT}$ provides sufficient image quality to replace MDCT for timely control after stent implantation. Because of the significant differences in the left and right branch diameter measurements between CBCT and MDCT, CBCT guidance is not recommended for conventional stent-graft planning. However, we believe that CBCT has a potential advantage as an imaging modality for patients in extremely urgent or weak condition. In our study, 5 patients with a choking risk or cachexy underwent stent-graft planning with СВCT guidance at the decision of the oncologist, respiratory specialist and interventional radiologist. These patients could be taken directly to the operating suite, where cross-sectional imaging can be performed without transferring them to the department of radiology, and the decision about airway stents could be made while preparing the patients.

In our study, airway stenting was successful on the first attempt in $90 / 100(90 \%)$ patients in the CBCTguided group and 123/128 (96\%) patients in the fluoroscopy-guided group. Although there was no significant difference, the ratios appeared to be different. In the CBCT-guided group, the interventional radiologist tended to adjust the stent position or introduce the balloon to expand the stent when its placement was not perfect. The patient outcomes and survival rates of the CBCT-guided group did not appear to be significantly different from those of the fluoroscopy-guided group $(P>0.05)$. However, in comparison with the fluoroscopy-guided group, the rate of major complications in the CBCT-guided group was significantly reduced $(P=0.010)$. This approach, especially 3D volume reconstruction technology, has particular advantages in minimizing migration after tracheal airway stent placement. 
To the best of our knowledge, little research has been presented regarding the application of CBCT during airway stent placement. The technical image quality of CBCT is inferior to that of MDCT. ${ }^{[14]}$ However, clinical experience from other centers has demonstrated the usefulness of CBCT imaging for the detection of complications in various medical contexts, such as during neuro-endovascular procedures,

$[13,15-20]$ showing that it can improve clinical outcomes. ${ }^{[21]}$ A new study from Guowu Zhou and his colleagues showed that CBCT can provide 3-dimensional images that enable accurate positioning of the cryoprobe for transbronchial cryobiopsy and interstitial lung disease as well as reduce the risks of pneumothorax and massive bleeding. ${ }^{[22]}$ In our study, we came to the same conclusion that intraoperative $\mathrm{CBCT}$ decreased the rates of major complications, especially stent migration.

Study limitations

The current study has some limitations. First, this is a retrospective study with inevitable selection bias. Second, although this work has a relatively large sample size, it is limited by the lack of a randomized controlled design, which may weaken the statistical power of our results. However, in this study cohort, the locations of airway diseases covered a wide range. Thus, our finding of statistical significance should be credible. In addition, the process of operation may increase the radiation exposure of interventional radiologists. Nevertheless, we strictly monitored the radiation exposure time and always strive to improve the clinical prognosis of patients.

\section{Conclusion}

In summary, CBCT imaging is of sufficient quality to replace MDCT for timely control after stent implantation and for stent-graft planning in some cases; it also reduces the complications of airway stent placement. In our opinion, a CBCT apparatus might also represent a valuable adjunct to the standard equipment that is normally available in an operating room for airway stent procedures.

\section{Declarations}

Statement of Ethics: Written informed consent was obtained from each patient for the publication of this article and any accompanying images. This study was approved by the Ethics Committee of Zhengzhou University. The procedures followed were in accordance with the Helsinki Declaration of 1975, as revised in 1983.

Conflict of Interest Statement: The authors have no conflicts of interest to declare.

Author contributions: Jing.Li. Kaihao.Xu. Dechao.Jiao. and Xinwei.Han. wrote the main manuscript text and Zhaonan.Li. Xueliang.Zhou. Yahua.Li. and Juanfang.Liu. prepared figures 1-3. Zaoqu.Liu. and 
Yuan.Yao. prepared table1-7. All authors reviewed the manuscript.

Funding: This work was supported by the young and middle-aged health science and technology innovation talent project of Henan Province in 2020 (yxkc2020037); This work was supported by Henan Provincial Health commission and ministry jointly support the youth project (sb201902014)

\section{References}

1. Han X, Yin M, Li L, Zhu M, Ren K, Qi Y, Li X, Wu G. Customized airway stenting for bronchopleural fistula after pulmonary resection by interventional technique: single-center study of 148 consecutive patients. Surg Endosc. 2018 Oct;32(10):4116-4124.

2. Li Y, Zhou X, Ren K, Ren J, Han X. Bronchopleural Fistula Cured by Customized Airway Metallic Stent. Chest. 2019 Nov;156(5):1031.

3. Kim J, Shin JH, Kim JH, Song HY, Song SY, Park CK. Metallic stent placement for the management of tracheal carina strictures and fistulas: technical and clinical outcomes. AJR Am J Roentgenol. 2014 Apr;202(4):880-5.

4. Marchese R, Poidomani G, Paglino G, Crimi C, Lo Nigro C, Argano V. Fully covered self-expandable metal stent in tracheobronchial disorders: clinical experience. Respiration. 2015;89(1):49-56.

5. Saad CP, Murthy S, Krizmanich G, Mehta AC. Self-expandable metallic airway stents and flexible bronchoscopy: long-term outcomes analysis. Chest. 2003 Nov;124(5):1993-9.

6. Kim S, Gotway MB, Webb WR, Gordon RL, Golden JA. Tracheal compression by the stomach following gastric pull-up: diagnosis with CT and treatment with expandable metallic stent placement. Chest. 2002 Mar;121(3):998-1001.

7. Thornton RH, Gordon RL, Kerlan RK, LaBerge JM, Wilson MW, Wolanske KA, Gotway MB, Hastings GS, Golden JA. Outcomes of tracheobronchial stent placement for benign disease. Radiology. 2006 Jul;240(1):273-82.

8. Godoy MC, Saldana DA, Rao PP, Vlahos I, Naidich DP, Benveniste MF, Erasmus JJ, Marom EM, Ost D. Multidetector CT evaluation of airway stents: what the radiologist should know. Radiographics. 2014 Nov-Dec;34(7):1793-806.

9. Meyer BC, Frericks BB, Albrecht T, Wolf KJ, Wacker FK. Contrast-enhanced abdominal angiographic CT for intra-abdominal tumor embolization: a new tool for vessel and soft tissue visualization. Cardiovasc Intervent Radiol. 2007;30(4):743-749.

10. Li YD, Han XW, Li MH, Wu G. Bronchial stump fistula: treatment with covered, retrievable, expandable, hinged stents-preliminary clinical experience. Acta Radiol. 2006;47(9):922-926.

11. Bongartz G, Bongartz G, Geleijns J. European guidelines on quality criteria for computed tomography. Luxembourg: Office for Official Publications of the European Communities;1999 
12. Landis JR, Koch GG. The measurement of observer agreement for categorical data. Biometrics. 1977;33(1):159-174.

13. Eide KR, Ødegård A, Myhre HO, Lydersen S, Hatlinghus S, Haraldseth O. DynaCT during EVAR-a comparison with multidetector CT. Eur J Vasc Endovasc Surg. 2009;37(1):23-30.

14. Smyth J, Sutton D, Houston J. Evaluation of the quality of CT-like images obtained using a commercial flat panel detector system. Biomed Imaging Interv J. 2006;2(4):e48.

15. Biasi L, Ali T, Hinchliffe R, Morgan R, Loftus I, Thompson M. Intraoperative DynaCT detection and immediate correction of a type la endoleak following endovascular repair of abdominal aortic aneurysm. Cardiovasc Intervent Radiol. 2009;32(3):535-538.

16. Gupta V, Chugh M, Walia BS, Vaishya S, Jha AN. Digital subtraction angiography laboratory with inbuilt CT (DynaCT): application during intracranial anurysm embolization. Neurol India. 2008;56(1):90-91.

17. Wang CY, Xia JG, Chen WH, Lu YF, Han ZH, Wang Q. Value of Dyna CT in guiding embolization during transarterial uterine artery embolization of fibroids. Exp Ther Med. 2020;20(2):1353-1358.

18. Ritter M, Rassweiler MC, Michel MS. The Uro Dyna-CT Enables Three-dimensional Planned Laserguided Complex Punctures. Eur Urol. 2015;68(5):880-884.

19. Rotolo N, Floridi C, Imperatori A, Fontana F, lerardi AM, Mangini M, Arlant V, De Marchi G, Novario R, Dominioni L, Fugazzola C, Carrafiello G. Comparison of cone-beam CT-guided and CT fluoroscopyguided transthoracic needle biopsy of lung nodules. Eur Radiol. 2016 Feb;26(2):381-9.

20. Ashrafi-Asgarabad A, Safiri S. Prognostic Value of Primary Tumor Volume Changes on Kilovoltage Onboard Cone Beam Computed Tomography during Definitive Chemoradiotherapy for stage III NonSmall Cell Lung Cancer: Methodological Issues. J Thorac Oncol. 2018 Feb;13(2):e26-e27.

21. Li TF, Ma J, Han XW, Fu PJ, Niu RN, Luo WZ, Ren JZ. Application of High-Resolution C-Arm CT Combined with Streak Metal Artifact Removal Technology for the Stent-Assisted Embolization of Intracranial Aneurysms. AJNR Am J Neuroradiol. 2019 Oct;40(10):1752-1758. doi: 10.3174/ajnr.A6190. Epub 2019 Sep 26. PMID: 31558505; PMCID: PMC7028573.

22. Zhou G, Ren Y, Li J, Yang T, Su N, Zhao L, Wang D, Li Y, Tian Z, Liu R, Dai H, Wang C. Safety and diagnostic efficacy of cone beam computed tomography-guided transbronchial cryobiopsy for interstitial lung disease: a cohort study. Eur Respir J. 2020 Aug 6;56(2):2000724.

\section{Tables}

Table1 Baseline Characteristics 


\begin{tabular}{|c|c|c|c|}
\hline Characteristic & $\begin{array}{l}\text { CBCT-guided } \\
(n=100)\end{array}$ & $\begin{array}{l}\text { Fluoroscopy- } \\
\text { guided } \\
(n=128)\end{array}$ & $\begin{array}{l}P \\
\text { Value }\end{array}$ \\
\hline \multicolumn{4}{|l|}{ Age $(y)$} \\
\hline Mean/ Range & $59.6 \pm 13.6(16-89)$ & $60.1 \pm 12.7(21-90)$ & $.304^{*}$ \\
\hline Sex & & & $.810^{\dagger}$ \\
\hline Male & 71 & 89 & \\
\hline Female & 29 & 39 & \\
\hline Disease & & & $.388^{\dagger}$ \\
\hline Stenosis & 63 & 91 & \\
\hline Fistula & 34 & 35 & \\
\hline Both & 3 & 2 & \\
\hline Etiology & & & $.606^{\#}$ \\
\hline Esophageal cancer & 52 & 66 & \\
\hline Lung cancer & 21 & 27 & \\
\hline Mediastinal tumor & 11 & 9 & \\
\hline Thyroid cancer & 5 & 10 & \\
\hline Tuberculosis & 3 & 7 & \\
\hline Lung infection & 2 & 6 & \\
\hline Tracheal tube injury & 2 & 2 & \\
\hline Neck trauma & 2 & 1 & \\
\hline Other & 2 & 0 & \\
\hline location of diseases & & & $.541^{\dagger}$ \\
\hline Trachea & 59 & 71 & \\
\hline Right bronchus & 12 & 19 & \\
\hline Left bronchus & 17 & 28 & \\
\hline Carina & 12 & 10 & \\
\hline Types of Stents & & & $.660^{\dagger}$ \\
\hline Y-shaped covered metallic stent & 52 & 68 & \\
\hline
\end{tabular}




\begin{tabular}{|lll|}
\hline Tubular covered metallic stent & 31 & 42 \\
\hline Tubular uncovered metallic stent & 14 & 11 \\
\hline $\begin{array}{l}\text { Y-shaped uncovered } \\
\text { metallic stent }\end{array}$ & 2 & 4 \\
\hline L-shaped covered metallic stent & 1 & 3 \\
\hline Comorbid disease & 21 & \\
\hline Hypertension & 9 & 28 \\
\hline Diabetes & 7 & 12 \\
\hline Coronary heart disease & 7 & $996^{\dagger}$ \\
\hline $\begin{array}{l}\text { Renal insufficiency (serum creatinine }>140 \\
\text { mmol/L) }\end{array}$ & 5 & 11 \\
\hline hepatic insufficiency & & $7(3-11)$ \\
\hline Hospitalization & $5(3-7)$ & $973^{\ddagger}$ \\
\hline Median/ Range & 5 & \\
\hline
\end{tabular}

Note. - Numbers in parentheses are the range. $S D=$ standard deviation.

*Student $t$ test

${ }^{\dagger}$ Pearson $\chi 2$ test

${ }^{\ddagger}$ Wilcoxon signed rank test

\#The Fisher exact test was used.

Table2 Comparing CBCT and MDCT. Measured lengths and diameters in $\mathrm{mm}( \pm \mathrm{SD})$ 


\begin{tabular}{|llll|}
\hline Stent graft measurement & CBCT $₫ \mathrm{n}=200 \rrbracket$ & MDCT $(\mathrm{n}=200)$ & $P$ Value \\
\hline Stent length & $58.7( \pm 16.82)$ & $58.5( \pm 17.06)$ & 0.883 \\
\hline Upper diameter & $18.2( \pm 2.81)$ & $19.0( \pm 2.33)$ & 0.161 \\
\hline Bottom diameter & $18.2( \pm 2.77)$ & $18.8( \pm 2.65)$ & 0.161 \\
\hline Left branch length & $24.7( \pm 8.86)$ & $24.4( \pm 8.42)$ & 0.223 \\
\hline Left branch diameter & $13.3( \pm 3.79)$ & $13.5( \pm 3.32)$ & 0.019 \\
\hline Right branch length & $16.9( \pm 7.81)$ & $17.2( \pm 7.42)$ & 0.563 \\
\hline Right branch diameter & $12.9( \pm 2.05)$ & $13.2( \pm 1.91)$ & 0.023 \\
\hline
\end{tabular}

N⿴囗The total number of observations made for each measurement. For example, 200 for CBCT means 100 patients all observed by two radiologists

Table3 Comparing CBCT and MDCT. Score-points on a scale from 1-4 for visibility of anatomical areas $( \pm S D)$

\begin{tabular}{|lllc|}
\hline Anatomical ares & CBCT & MDCT & PValue \\
\hline Is the whole stent-graft recorded in the volume? & $3.7( \pm 0.48)$ & $4.0( \pm 0.12)$ & $.178^{\ddagger}$ \\
\hline Markers in the stent graft: number and positions are assessed & $3.6( \pm 0.59)$ & $3.9( \pm 0.28)$ & $.180^{\ddagger}$ \\
\hline Airway above upper stent graft & $3.8( \pm 0.41)$ & $4.0( \pm 0.20)$ & $.001^{\ddagger}$ \\
\hline Airway under bottom stent graft & $3.6( \pm 0.56)$ & $3.9( \pm 0.24)$ & $.167^{\ddagger}$ \\
\hline Bifurcation & $3.6( \pm 0.53)$ & $4.0( \pm 0.17)$ & $.253^{\ddagger}$ \\
\hline Distal bronchus & $3.7( \pm 0.49)$ & $3.9( \pm 0.34)$ & $.001^{\ddagger}$ \\
\hline Pulmonary parenchyma & $3.3( \pm 0.71)$ & $3.9( \pm 0.31)$ & $.000^{\ddagger}$ \\
\hline
\end{tabular}

‡Wilcoxon signed rank test. 
Table4 Intraobserver variability in Stent graft measurement

\begin{tabular}{|ll|}
\hline 1.Stent graft measurement & $\mathrm{ICC}(95 \llbracket \mathrm{Cl})$ \\
\hline 2.Stent length & $0.998(0.996-0.999)$ \\
\hline 3.Upper diameter & $0.849(0.776-0.899)$ \\
\hline 4.Bottom diameter & $0.861(0.726-0.922)$ \\
\hline 5.Left branch length & $0.988(0.979-0.993)$ \\
\hline 6.Left branch diameter & $0.943(0.883-0.970)$ \\
\hline 7.Right branch length & $0.992(0.975-0.997)$ \\
\hline 8.Right branch diameter & $0.812(0.672-892)$ \\
\hline
\end{tabular}

Table5 Intraobserver variability in the visibility of anatomical areas 
A.Is the whole stent-graft recorded in the volume? 4-yes with good margin; 3-just barely; $2-<10 \%$ is missing and $1->10 \%$ is missing

$0.776(0.628-$

B.Markers in the stent graft: number and positions are assessed $0.9)$

$0.824(0.711-$ $0.934)$

C.Airway above upper stent graft 0.692(0.567$0.814)$

D.Airway under bottom stent graft $0.652(0.494-$ $0.786)$

E.Bifurcation $0.687(0.531-$ $0.822)$

F.Distal bronchus $0.646(0.497-$ $0.772)$

G.Pulmonary parenchyma 0.673(0.533$0.796)$

Table6 Summary of Complications 


\begin{tabular}{|c|c|c|c|}
\hline Complications & $\begin{array}{l}\text { CBCT- guided } \\
(n=100)\end{array}$ & $\begin{array}{l}\text { Fluoroscopy- guided } \\
(n=128)\end{array}$ & $\begin{array}{l}P \\
\text { Value }\end{array}$ \\
\hline Major complications & $7(0.07)$ & $24(0.19)$ & $.010^{\dagger}$ \\
\hline Migration & $3(0.03)$ & $13(0.10)$ & $.036^{\dagger}$ \\
\hline Sputum retention & $2(0.02)$ & $5(0.04)$ & $.471^{\#}$ \\
\hline Segmental atelectasis & $1(0.01)$ & $3(0.02)$ & $.633^{\#}$ \\
\hline Pneumothorax & $1(0.01)$ & $3(0.02)$ & $.633^{\#}$ \\
\hline Minor complications & $14(0.14)$ & $23(0.18)$ & $.420^{\dagger}$ \\
\hline Slight hemoptysis & $7(0.07)$ & $9(0.07)$ & $.993^{\dagger}$ \\
\hline Chest or throat pain & $4(0.04)$ & $5(0.04)$ & $.971^{\dagger}$ \\
\hline Laryngeal foreign body sensation & $2(0.02)$ & $2(0.02)$ & $\nabla 0.99^{\#}$ \\
\hline Eating disorder & $1(0.01)$ & $3(0.02)$ & $.633^{\#}$ \\
\hline
\end{tabular}

Data are numbers of events. Data in parentheses are percentages.

${ }^{\dagger}$ Pearson $\chi 2$ test

\#The Fisher exact test was used.

Table7 Patient outcome of airway stent placement in two groups

\begin{tabular}{|llll|}
\hline patient outcome & CBCT- guided & Fluoroscopy- guided & P Value \\
\hline death & $38(0.38)$ & $51(0.398)$ & $.777 \dagger$ \\
\hline with improvement & $47(0.47)$ & $51(0.398)$ & $.279 \dagger$ \\
\hline cure & $12(0.12)$ & $19(0.148)$ & $.534 \dagger$ \\
\hline lost to follow-up & $3(0.03)$ & $7(0.054)$ & $.366 \dagger$ \\
\hline
\end{tabular}

tPearson $\chi 2$ test 
Figures

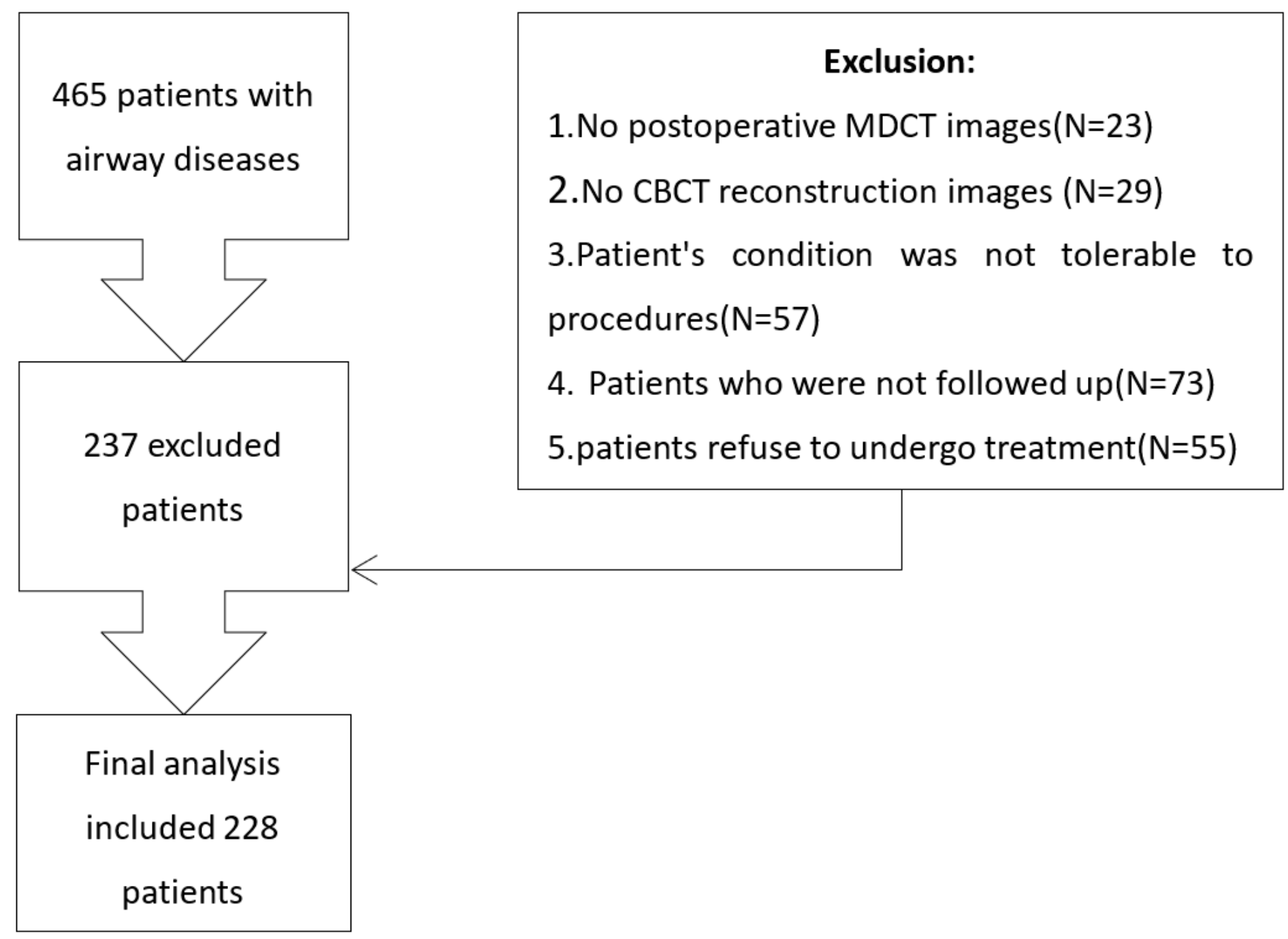

Figure 1

Flow diagram of the case selection procedure. 


\section{Survival proportions: Survival of Two groups}

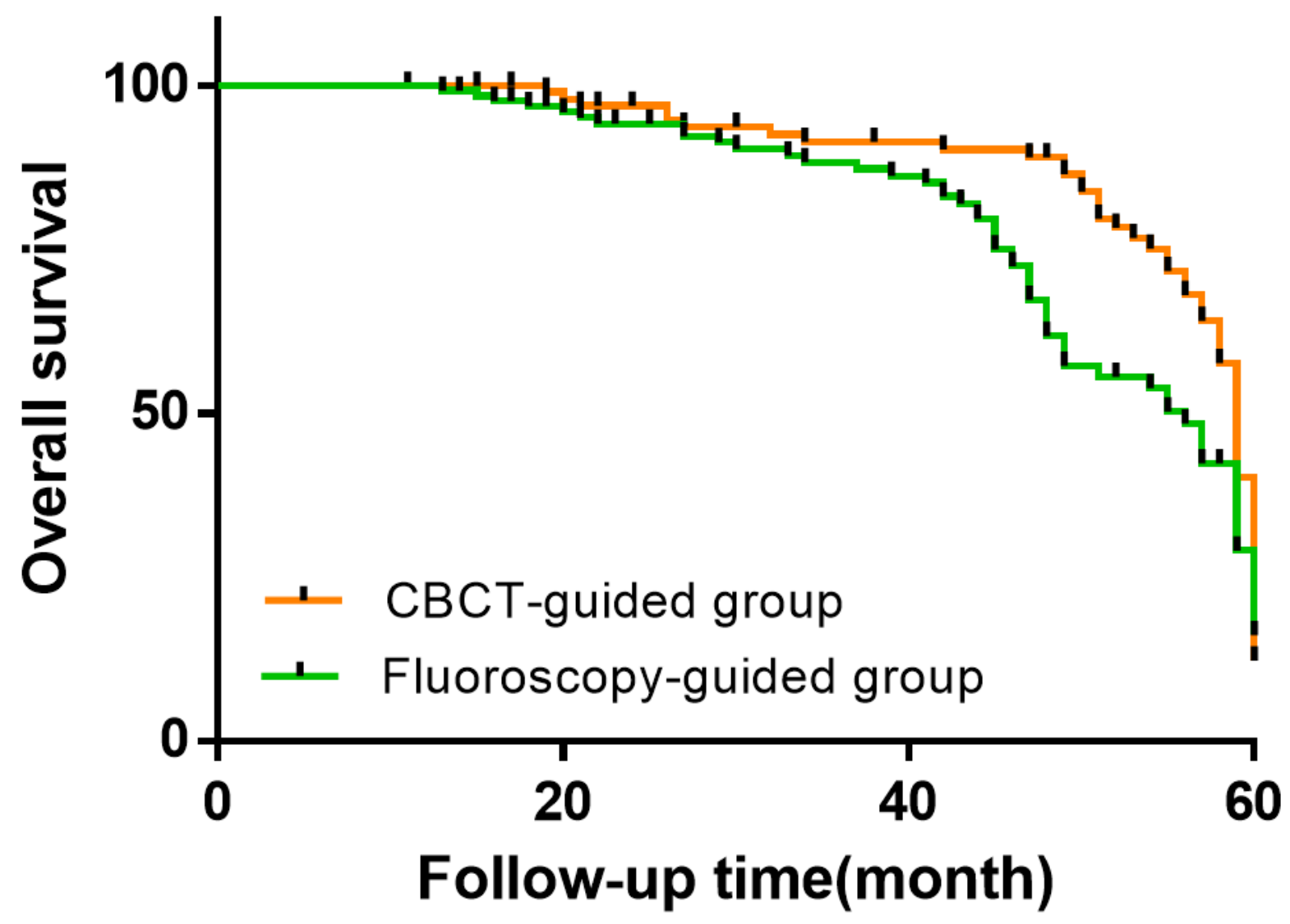

Figure 2

Kaplan-Meier the overall survival (OS) with CBCT-guided group versus Fluoroscopy- guided group. Mean OS was 44.320 months $( \pm 15.45)$ versus 41.007 months $₫ \pm 14.5 \rrbracket)$ ( $p=$, log-rank test). The 1-, 3- and 5-year overall survival rates of CBCT-guided group were $100.0 \%, 72.4 \%$ and $61.0 \%$ respectively, while those of Fluoroscopy-guided group were $100.0 \%, 69.8 \%$, and $57.0 \%$ respectively(p>.05) 

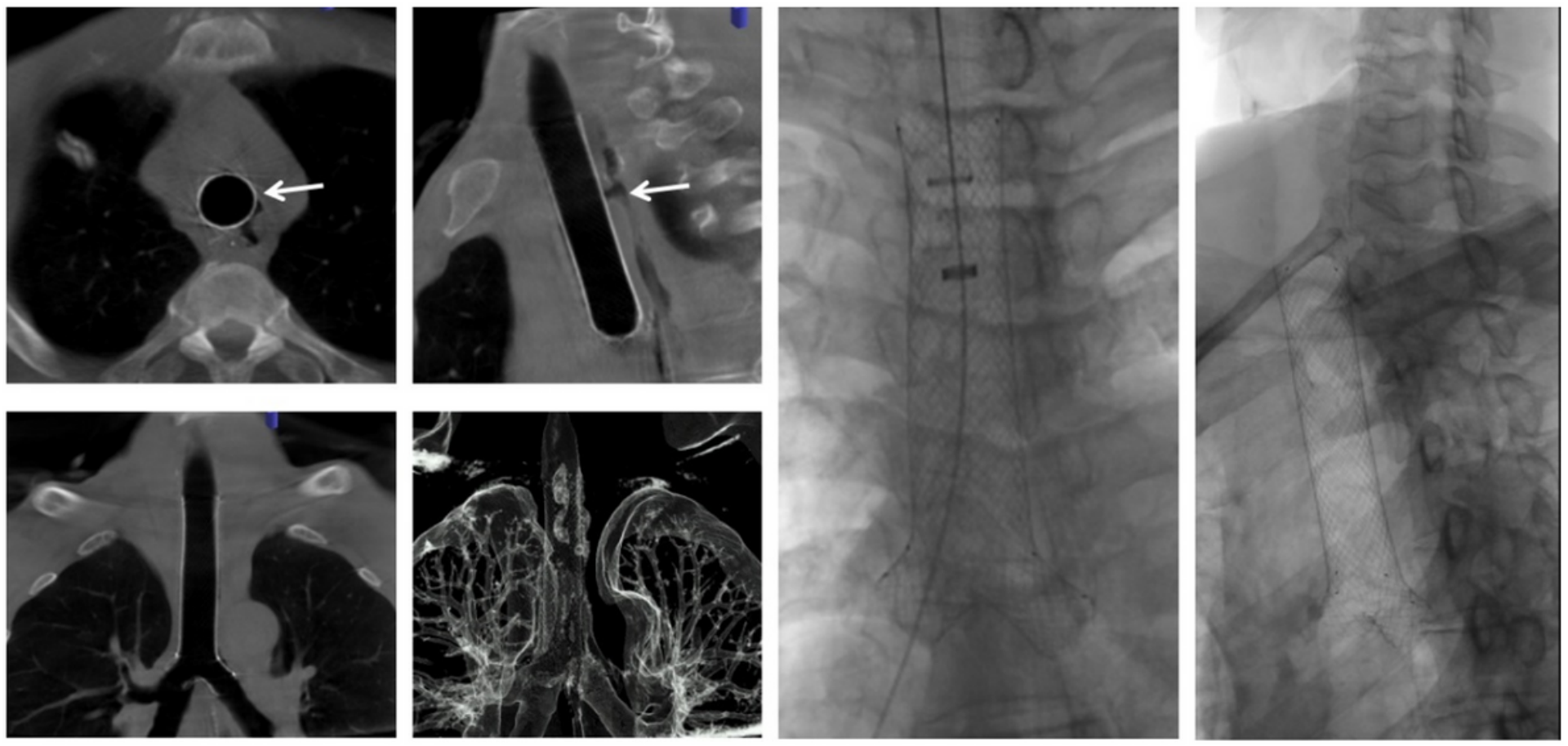

Figure 3

A 67-year-old male with an airway fistula treated with stents. (a, b, c) After airway fistula in the main bronchus was treated with a large y-covered stent, use the CBCT to confirm stent deployment and apposition in the cross-section, sagittal-section and coronal-section. (d) CBCT 3-D reconstruction shows tracheal tree morphology after stent implantation. $(e, f)$ the images of Fluoroscopy-guided airway stent procedures. 